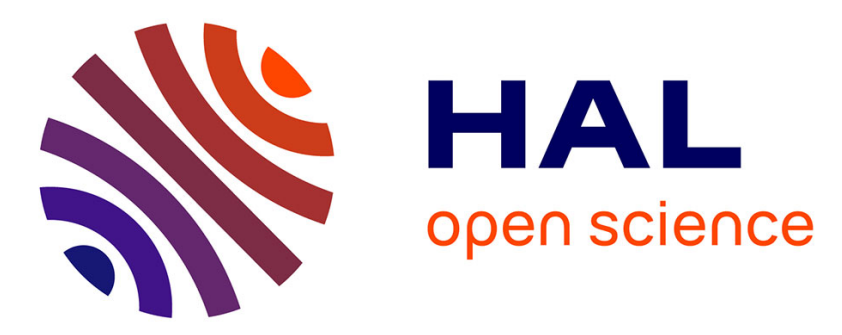

\title{
Tracing Imperfectly Modular Variability in Software Product Line Implementation
}

Xhevahire Tërnava, Philippe Collet

\section{To cite this version:}

Xhevahire Tërnava, Philippe Collet. Tracing Imperfectly Modular Variability in Software Product Line Implementation. The 16th International Conference on Software Reuse, May 2017, Salvador de Bahia, Brazil. hal-01486216

\section{HAL Id: hal-01486216 https://hal.science/hal-01486216}

Submitted on 9 Mar 2017

HAL is a multi-disciplinary open access archive for the deposit and dissemination of scientific research documents, whether they are published or not. The documents may come from teaching and research institutions in France or abroad, or from public or private research centers.
L'archive ouverte pluridisciplinaire HAL, est destinée au dépôt et à la diffusion de documents scientifiques de niveau recherche, publiés ou non, émanant des établissements d'enseignement et de recherche français ou étrangers, des laboratoires publics ou privés. 


\title{
Tracing Imperfectly Modular Variability in Software Product Line Implementation
}

\author{
Xhevahire Tërnava and Philippe Collet \\ Université Côte d'Azur, CNRS, I3S, France \\ \{ternava, collet\}@i3s.unice.fr
}

\begin{abstract}
When large software product lines are engineered, a combined set of traditional techniques, e.g., inheritance, design patterns, generic types, is likely to be used for realizing the variability at the implementation level. In these techniques the concept of feature, as a reusable unit, does not have a first-class representation in implementation, but still an imperfect form of modularization of variability can be achieved. We present in this paper a framework $(i)$ to explicitly capture and document this imperfectly modular variability - by several combined techniques - in a dedicated variability model, and ( $i i)$ to establish trace links between this model and the variability model at the specification level. We report on the implementation of the framework through a domain specific language, and show the feasibility of the approach on a real feature-rich system.
\end{abstract}

\section{Introduction}

In Software Product Line Engineering (SPLE), the core software assets are developed during the domain engineering phase 13 and represent those reusable artifacts and resources that form the basis for eliciting the single software products during the application engineering phase. Core assets are made reusable by modeling and realizing what is common and what is going to vary, i.e., the commonality and the variability, between the related products in a methodological way. In realistic SPLs, where variability is extensive, a crucial issue is the ability to manage it in these different core assets among different abstraction levels [5]. An important aspect of the variability management activity is then the ability to trace a variable unit, commonly known as a feature [10], along the SPLE phases.

Traceability is defined as the ability to describe and follow the life of a software artifact forward and backward along the software lifecycle [2]. In SPLE there are four main dimensions of traceability: refinement, similarity, variability, and versioning traceability 2, 13. Variability traceability "is dealt by capturing variability information explicitly and modeling the dependencies and relationships separate from other development artifacts" [4]. In this work we focus on an analysis of the variability traceability between the specification and implementation level, i.e., on the realization trace links [2,13] to the core-code assets.

The results of a recent survey on SPLE traceability, by Kim et al. [12], show that none of the current approaches fully support end-to-end traceability, 
and there are unexplored research areas in these approaches. To the best of our knowledge, no variability traceability and management approache at the implementation level, e.g., $3,4,6,9,13,14$, , is currently addressing the early steps of capturing and modeling the variability when a subset of traditional techniques (e.g., inheritance, design patterns, generic types) are used in combination, as in many realistic SPL settings $3,8,8,9]$.

In this paper, we propose an approach for tracing variability between the specification level and, what we name imperfectly modular variability 1 at the implementation level. We distinguish both levels in a way similar to Becker [3] Our contribution is a tooled framework (Sec. 3) that gives support from the early steps of variability traceability, i.e., from the capturing and modeling of the variability in core-code assets. Unlike other works that tackled similar issues $[6,9,14]$ we show how to capture and model the variability when it is implemented by several traditional techniques in combination. We keep the variability information separated from the core code assets as by Berger et al. [4] and Pohl et al. 13. Further, as the variability to be represented may be large [13, our framework fosters the documentation of the implemented variability in a fragmented and flexible way, inspired by Kästner et al. [11. We also report on our implementation of the framework by a Domain Specific Language (DSL), and on its application to a feature-rich system, showing its feasibility (Section 4).

\section{Motivations}

Background. We consider that the variability of an SPL is documented in a Variability Model (VM), which is commonly expressed as a Feature Model (FM) [10]. An FM is mainly used at the specification level for scoping the software products within an SPL in terms of features. On the other hand variation points $(v p$-s) are places in a design or implementation that identify locations at which the variation occurs [9], and the way that a $v p$ is going to vary is expressed by its variants.

Variability traceability can be established and used for different reasons, and by different stakeholders [2,7]. It is mainly used for (semi)automating different processes in SPLE, e.g., for resolving the variability (product derivation), evolving, checking consistency, addressing, or comprehending the variability.

Imperfectly Modular Variability. For illustration and validation we use JavaGeom 2 an open source geometry library for Java that is architected around well identified features. Although not presented as an SPL, JavaGeom is a relevant and easily understandable case for demonstrating the applicability of our framework on the equivalent of a medium-size SPL. Let us consider the implementation of a set of features as depicted in the FM on Fig. 11 StraightCurve2D is a mandatory feature with three shown alternative features: Line2D, Segment2D, and Ray2D. Focusing on the realization techniques ( $c f$. Fig. 2), the abstract class

\footnotetext{
1 This notion is defined in Sec. 2 using a real feature-rich system.
}

2 http://geom-java.sourceforge.net/index.html 


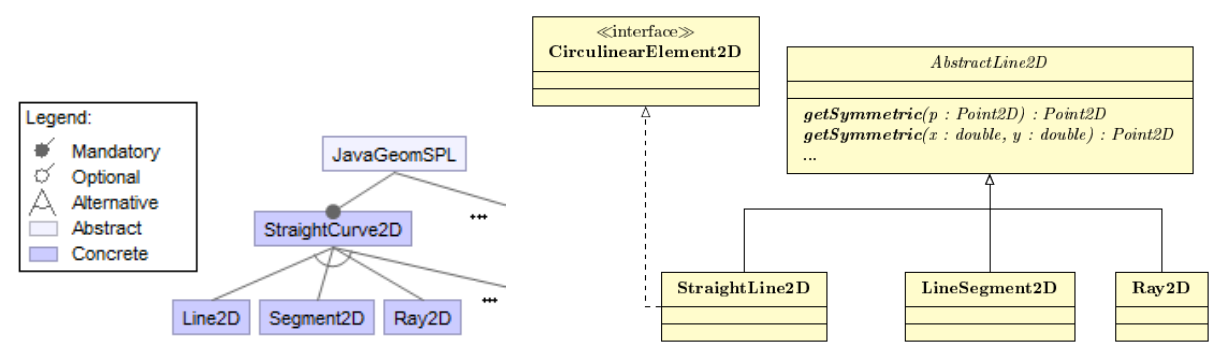

Fig. 1: Features from JavaGeom

Fig. 2: A detailed design excerpt of JavaGeom

AbstractLine2D is a $v p$ and its three variants, i.e., StraightLine2D, LineSegment2D, and Ray2D, are created by generalizing/specializing its implementation. Features from Fig. 1 seem to have a direct and perfect modular mapping in implementation (cf. Fig. 2), e.g., «StraightCurve2D implemented by AbstractLine2D», or «Line2D implemented by StraightLine2D». But actually, this perfect modularity hardly exists.

Imperfect modularity comes from the fact that a feature is a domain concept and its refinement in core-code assets is a set of $v p$-s and variants (even if they are modular), i.e., it does not have a direct and single mapping. For example, the feature Line2D uses several $v p$-s, such as AbstractLine2D and CirculinearElement2D (other $v p$-s are not shown), the variant StraightLine2D ( $c f$. Fig. 2), plus their technical $v p$-s (Sec. 3.2), as the getSymmetric() $v p$ of the AbstractLine2D. When a combined set of traditional techniques are used for implementing the variability, e.g., inheritance for StraightCurve2D, overloading for getSymmetric(), the code is not shaped in terms of features. Therefore, the trace relation is $n-t o-m$ between the specified features to the $v p$-s and variants at the implementation. Moreover an SPL architect has to deal with a variety of $v p$-s and variants.

Our approach addresses the ability to trace this imperfectly modular variability in implementation, which we define as follows: An imperfectly modular variability in implementation occurs when some variability is implemented in a methodological way, with several implementation techniques used in combination, the code being not necessarily shaped in terms of features, but still structured with the variability traceability in mind.

\section{A Three Step Traceability Approach}

To trace imperfectly modular variability in implementation, we propose an approach that follows three main steps ( $c f$. Fig. 3): 1) capturing the implemented variability in terms of $v p$-s and variants, as abstract concepts, (2) modeling (documenting) the variability in terms of $v p$-s and variants, while keeping the consistency with their implementation in core-code assets, and (3) establishing the trace links between the specified and implemented variabilities. 


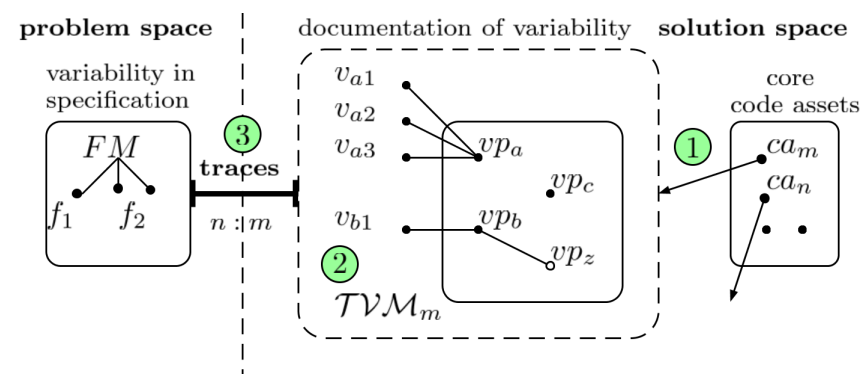

Fig. 3: Proposed traceability approach $\left(\mathcal{T} \mathcal{V} \mathcal{M}_{m}\right.$ stands for Technical Variability Model (cf. Sec. 3.2) of the core-code asset $c a_{m}$, with $v p-s\left\{v p_{a}, v p_{b}, \ldots\right\}$ and their respective variants $\left\{v_{a 1}, v_{a 2}, \ldots\right\}$. While, $\left\{f_{1}, f_{2}, \ldots\right\}$ are features in the FM.)

\subsection{Capturing the Variability of Core-Code Assets (Step (1)}

The core-code assets that realize variability usually consist of a common part and a variable part. It can happen that a whole asset is also a variable asset. A core-code asset can be, e.g., a source file, package, class. The variable part consists of a mechanism (i.e., technique) for creating the variants, a way for resolving the variants, and the variants themselves. They are abstracted using the concepts of variation points ( $v p-\mathrm{s})$, variants, and their dependencies.

Let the set of all $v p$-S in an SPL be $\mathcal{V P}=\left\{v p_{a}, v p_{b}, v p_{c}, \ldots\right\}$ for the set of core-code assets, with variability or variable, $\mathcal{C} \mathcal{A}=\left\{c a_{m}, c a_{n}, c a_{o}, \ldots\right\}$. We assume that a $v p_{x} \in \mathcal{V} \mathcal{P}$ is implemented by a single traditional technique $t_{x} \in \mathcal{T}$. The set $\mathcal{T}$ of possible techniques for $v p_{x}$ is then made explicit in our framework,

$\mathcal{T}=\{$ Inheritance, Generic Type, Overriding, Strategy

pattern, Template pattern,...

A $v p$ is not a by-product of an implementation technique [5], therefore we have to tag it in some way. "Tagging a $v p$ " means to map the $v p_{x} \in \mathcal{V} \mathcal{P}$ concept to its concrete varying element of any $c a_{x} \in \mathcal{C} \mathcal{A}$. For example, we abstract/tag the superclass AbstractLine2D ( $c f$. Fig. 2) with vp_AbsLine2D, and its subclasses as variants v_Line2D, v_Segment2D, and v_Ray2D, respectively. Depending on the size of variability and the used technique, the nature of a core asset element that represents a $v p$ varies. We gathered their variety as characteristic properties of $v p$-s. Their properties that are important to be captured are: granularity, relation logic, binding time, evolution, and resolution.

Granularity. A $v p$ in a core-code asset can represent a coarse-grained element that is going to vary, e.g., a file, package, class, interface; a medium-grained element e.g., a method, a field inside a class; or a fine-grained element e.g., an expression, statement, or block of code.

Logic Relation $(\mathcal{L G})$. The set of the logic relations between variants in a $v p$, that are commonly faced in practice, is similar to the possible relations between features in an FM. Thus, a $t_{x} \in \mathcal{T}$ offers at least one of these logic relations,

$\mathcal{L G}=\{$ Mandatory, Optional, Alternative, Multi-Coexisting $\}$ 
Binding Time $(\mathcal{B} \mathcal{T})$. Each $v p$ is associated with a binding time, i.e., the time when is decided for its variants, or when they are resolved. Based on the available taxonomies [6], the possible binding times for the $v p_{x}$ are:

$\mathcal{B T}=$ Compilation, Assembly, Programming time,

Configuration, Deploy, StartUp, Runtime\}

Evolution $(\mathcal{E} \mathcal{V})$. Depending on whether a $v p$ is meant to be evolved in the future with new variants or not, it can be $\mathcal{E} \mathcal{V}=\{$ Open, Close .

For example, the vp_AbsLine2D has a class level granularity ( $c f$. Fig. 2). It is resolved at runtime to one of its alternative variants, v_Line2D, v_Segment2D, or v_Ray2D, and we tag it as open as it is implemented as an abstract class.

Another characteristic property of a $v p$ is how it is resolved, i.e., whether a variant is added, removed or replaced by another variant. This matters during the process of product derivation, which is a possible usage of our framework.

\subsection{Modeling the Implemented Variability (Step (2)}

During this phase, the variability of a core-code asset $c a_{x}$ is modeled in terms of $v p$-s and variants as abstractions ( $c f$. Fig. 3. step (2)). We distinguish five types of $v p$-s that can be chosen from:

$\mathcal{X}=\left\{v p, \quad v p \_u n i m p l ., v_{\text {_technical, }}\right.$ vp_optional, vp_nested $\}$

A resolution for each of them is given in Tbl. 1 . The implementation technique $t_{x} \in \mathcal{T}$ of the $v p_{x} \in \mathcal{V} \mathcal{P}$, which relation we write as $\left(v p_{x}, t_{x}\right)$, describes three main properties of the $v p_{x}$ : the relation logic for its variants, the evolution, and the binding time. Possibly, other properties can also be abstracted and attached. Then, $t_{x}=\left\{l g_{x}, e v_{x}, b t_{x}, \ldots\right\}$, where $l g_{x} \in \mathcal{L G}, e v_{x} \in \mathcal{E} \mathcal{V}$, and $b t_{x} \in \mathcal{B} \mathcal{T}$. So, when the $v p_{x}$ is an ordinary $v p(c f$. Tbl. 1) we model the variability in a core-code asset as a set of its variants $\mathcal{V}=\left\{v_{x 1}, v_{x 2}, v_{x 3}, \ldots\right\}$, and the characteristic properties derived from the $v p_{x}$ 's implementation technique $t_{x}$. This leads to the following definition:

$$
v p_{x}=\left\{\mathcal{V}, t_{x}\right\}=\left\{\left\{v_{x 1}, v_{x 2}, v_{x 3}, \ldots\right\}, t_{x}\right\}
$$

As an illustration we present the ordinary $v p_{x}$ (cf. Tbl. 11) as in Fig. 4 numbered with 1 . Similarly, a technical $v p, e . g$. , the technical $v p_{a}$ of $v p_{y}$, is represented as in 2. An optional $v p$ is modeled as the $v p_{z}$ in 3 . We use the acronym opt here in order to distinguish between the optional relations between variants in a $v p$ and the optionality of the $v p$ itself. Moreover a nested $v p$ is illustrated as the nested $v p_{b}$ of $v p_{z}$, where the variant $v_{z 2}$ of $v p_{z}$ represents a common part for its three variants $\left\{v_{b 1}, v_{b 2}, v_{b 3}\right\}$. Finally, the $v p_{c}=\left\{\{\varnothing\}, t_{c}\right\}$ in 4 is an unimplemented $v p$. These types of $v p$-s can be combined, e.g., an optional $v p$ can be ordinary, unimplemented, or can have other nested or technical $v p$-s.

Instead of modeling the whole implemented variability at once and in one place, we model it in a fragmented way. A fragment can be any unit (i.e., a package, a file, or a class) that has its inner variability and that is worth to be separately modeled. For this reason, we designed specific models, named as 
Table 1: Types of variation points that are commonly faced in practice

\begin{tabular}{ll}
\hline Types & Description \\
\hline Ordinary & A vp is introduced and implemented (i.e., its variants are realized) \\
& by a specific technique. \\
Unimplemented & A vp is introduced but is without predefined variants (i.e., its vari- \\
& ants are unknown during the domain engineering). \\
Technical & A vp is introduced and implemented only for supporting internally \\
& the implementation of another vp, which realizes some of the vari- \\
& ability at the specification level. \\
Optional & The vp itself, not its variants, is optional (i.e., when it is included \\
Nested $v p$ & or excluded in a product, so are its variants). \\
& When some variable part in a core-code asset becomes the common \\
& part for variants.
\end{tabular}

Technical Variability Models $(\mathcal{T V} \mathcal{M})$, which are created and maintained locally, i.e., closer to the core-code assets ( $c f$. Fig. 3). They contain the abstractions of $v p$ $\mathrm{s}$ and variants, their tags with core asset elements, and describe the variability of a specific core-code asset. For example, the variability of a $c a_{m}$ with six different $v p$-s is modeled by the $\mathcal{T} \mathcal{V} \mathcal{M}_{m}$ as in Fig. 4

All the $\mathcal{T} \mathcal{V} M s$ together constitute the Main Technical Variability Model $(\mathcal{M T} \mathcal{V} \mathcal{M})$. Unlike the organization of features in an FM as a tree structure, in $\mathcal{M T} \mathcal{V M}$ the $v p$-s reside in a forest-like structure. Moreover, the meaning of a $v p$ is extended, i.e., it is the place at which the variation occurs [9], and represents the used technique to realize the variability.

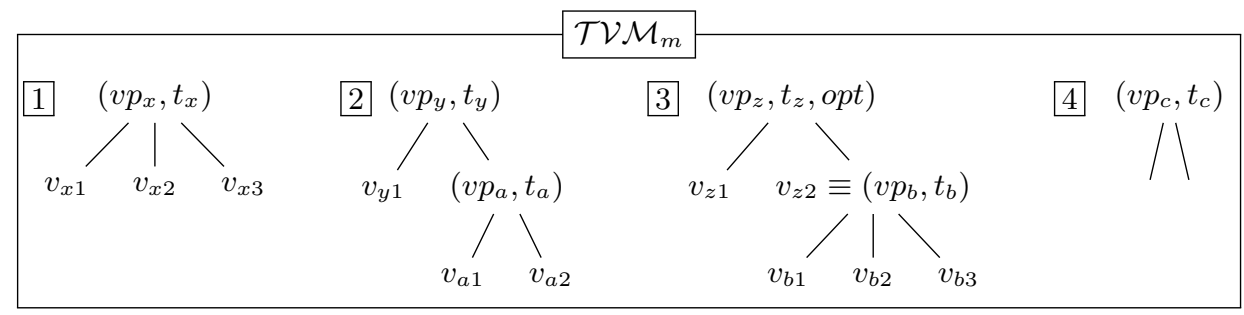

Fig. 4: An example of the Technical Variability Model $\left(\mathcal{T} \mathcal{V} \mathcal{M}_{m}\right)$ for a $c a_{m}$

\subsection{Establishing the Trace Links (Step (3)}

The last step of our approach ( $c f$. Fig. 3 step (3) is to map the variability between the VM at specification level (i.e., features in an FM) and the $\mathcal{M T V M}$ at implementation level (i.e., vp-s and variants), by establishing the trace links between them.

Let us suppose that $f_{x} \in \mathcal{F M}$ is a variable feature at specification level, where $\mathcal{F} \mathcal{M}=\left\{f_{1}, f_{2}, f_{3}, \ldots\right\}$. For mapping features to $v p$-s, we use a single bidirectional type of trace links implementedBy $(\longmapsto)$ or implements $(\longleftrightarrow)$, which 
presents the variability realization trace link in implementation. When $f_{x}$ is implemented ideally by a single variation point $v p_{x}$, or conversely, we write: $f_{x} \longmapsto v p_{x}$, or implementedBy $\left(f_{x}, v p_{x}\right)$. Similarly, $f_{x}$ can be implemented by a single variant $v_{x n} \in \mathcal{V}$ (cf. Sec. 3.2), i.e., $f_{x} \longmapsto v_{x n}$. For example, the feature StraightCurve2D ( $c f$. Fig. 1) is implemented by vp_AbsLine2D (cf. Fig. 2), i.e., StraightCurve2D $\longmapsto$ vp_AbsLine2D, or Ray2D $\longmapsto v_{-}$Ray2D. When $f_{x}$ is implemented by several $v p$-s, which can be from the same core-code asset or not, then $f_{x} \longmapsto\left\{v p_{x}, v p_{y}, v p_{z}, \ldots\right\}$.

The mapping between features and $v p$-s is a partial mapping, as some features in $\mathcal{F} \mathcal{M}$ are abstract features (i.e., do not require an implementation), or they can be deferred to be implemented later.

\section{Implementation and Application}

We implemented the proposed framework as an internal DSL in Scala. The interoperability between Java and Scala enabled us to use the DSL in JavaGeom. To document and trace variability, the DSL provides two modules fragment and traces, respectively. We used them to analyse $92 \%$ of the 35,456 lines of code from JavaGeom. The successful documentation phase resulted in $11 \mathcal{T V} \mathcal{M}_{s}$, all of them being at the package level. We observed that $v p$-s in JavaGeom are implemented using up to three techniques, inheritance, overloading, and generic types. Then, we established the trace links between the specified features ( $c f$. Fig. 1. which consist of 110 features extracted from its documentation) to the $v p$-s and variants in implementation ( $c f$. Fig. 2). We successfully traced $199 v p$-s, with 269 variants, showing the feasibility of our approach.

Capturing and documenting different types of $v p$-s and their implementation techniques during the variability traceability, as in JavaGeom, becomes important during the usage of trace links. For example, the relation logic between the variants in a $v p$ is needed to check the consistency between the variability at the specification and implementation level. Similarly, knowledge of the binding time of $v p$-s is necessary during the product derivation.

\section{Conclusion}

Tracing the variability between the specification and implementation levels is an important part of the development process in SPL engineering. At the implementation level, a combination of traditional variability implementation techniques

are actually used in many realistic SPLs, thus leading to a form of imperfectly modular variability in implementation. The key contribution of our approach is a three-step framework for capturing, documenting, and tracing this imperfectly modular variability, together with some DSL-based tool support.

A limitation of the DSL, but not of the framework itself, is that we could not apply it for tracing the variability at the finest granularity level, e.g., at the expression level, as our internal DSL in Scala uses reflection for tagging the variability. Although using reflection is not mandatory, this helps to keep the strong 
consistency between the abstractions of $v p$-s and variants with the core-code assets themselves. Although we used the DSL successfully in a real feature-rich system, we plan to extend this framework in supporting the documentation of the dependencies between the $v p$-s themselves and to integrate the DSL with another DSL that models the variability specifically at the specification level, such as FAMILIAR [1]. With these two extensions, we plan to apply our variability traceability framework to (semi)automated consistency checking of variability between the specification and implementation levels on several case studies.

\section{References}

1. Acher, M., Collet, P., Lahire, P., France, R.B.: FAMILIAR: a domain-specific language for large scale management of feature models. Science of Computer Programming (SCP) 78(6), 657-681 (2013)

2. Anquetil, N., Kulesza, U., Mitschke, R., Moreira, A., Royer, J.C., Rummler, A., Sousa, A.: A model-driven traceability framework for software product lines. Software \& Systems Modeling 9(4), 427-451 (2010)

3. Becker, M.: Towards a general model of variability in product families. In: Workshop on Software Variability Management. Editors Jilles van Gurp and Jan Bosch. Groningen, The Netherlands. http://www. cs. rug. nl/Research/SE/svm/proceedingsSVM2003Groningen. pdf. pp. 19-27 (2003)

4. Berg, K., Bishop, J., Muthig, D.: Tracing software product line variability: from problem to solution space. In: Proceedings of the 2005 annual research conference of the South African institute of computer scientists and information technologists on IT research in developing countries. pp. 182-191. South African Institute for Computer Scientists and Information Technologists (2005)

5. Bosch, J., Florijn, G., Greefhorst, D., Kuusela, J., Obbink, J.H., Pohl, K.: Variability issues in software product lines. In: Software Product-Family Engineering, pp. 13-21. Springer (2001)

6. Capilla, R., Bosch, J., Kang, K.C.: Systems and Software Variability Management. Springer (2013)

7. Cleland-Huang, J., Gotel, O., Zisman, A.: Software and systems traceability, vol. 2. Springer (2012)

8. Coplien, J.O.: Multi-paradigm Design for C+. Addison-Wesley (1999)

9. Jacobson, I., Griss, M., Jonsson, P.: Software reuse: architecture, process and organization for business success. ACM Press/Addison-Wesley Publishing Co. (1997)

10. Kang, K.C., Kim, S., Lee, J., Kim, K., Shin, E., Huh, M.: FORM: a feature-oriented reuse method with domain-specific reference architectures. Annals of Software Engineering 5(1), 143-168 (1998)

11. Kästner, C., Ostermann, K., Erdweg, S.: A variability-aware module system. In: ACM SIGPLAN Notices. vol. 47, pp. 773-792. ACM (2012)

12. Kim, J., Kang, S., Lee, J.: A comparison of software product line traceability approaches from end-to-end traceability perspectives. International Journal of Software Engineering and Knowledge Engineering 24(04), 677-714 (2014)

13. Pohl, K., Böckle, G., van der Linden, F.J.: Software product line engineering: foundations, principles and techniques. Springer Science \& Business Media (2005)

14. Schmid, K., John, I.: A customizable approach to full lifecycle variability management. Science of Computer Programming 53(3), 259-284 (2004) 